\title{
BOUNDED AND PERIODIC SOLUTIONS OF LINEAR AND WEAKLY NONLINEAR STOCHASTIC ITÔ SYSTEMS
}

UDC 519.21

\author{
O. M. STANZHITS'KII
}

\begin{abstract}
Conditions for the existence of solutions that are mean square bounded and periodic in $\mathbf{R}$ are obtained for linear and weakly nonlinear stochastic Itô systems by using the Green function of the linear part of the systems.
\end{abstract}

Let $(\Omega, F, \mathrm{E})$ be a complete probability space, and let a standard $m$-dimensional Wiener process $\{W(t): t \in \mathbf{R}\}$ be defined on $(\Omega, F, \mathrm{E})$. Assume that the components $W_{i}(t), i=1, \ldots, m$, of the Wiener process are jointly independent Wiener processes defined on $\mathbf{R}$.

Let $F_{t}, t \in \mathbf{R}$, be the $\sigma$-algebra generated by the sets of the form

$$
\left\{W\left(s_{2}\right)-W\left(s_{1}\right): s_{1} \leq s_{2} \leq t\right\} .
$$

Then $W(t)$ is measurable with respect to the flow $F_{t}$ and $W(t)-W(s)$ does not depend on $F_{s}$ for $s<t$.

Consider the following stochastic differential equation:

$$
d x=f(t, x) d t+g(t, x) d W(t),
$$

where $t \in \mathbf{R}, x \in \mathbf{R}^{n}$, and the column-function $f$ and $n \times m$ matrix $g$ are such that there exists a strongly unique solution of the equation.

An $n$-dimensional stochastic process $x(t)$ is called a solution of equation (1) in $\mathbf{R}$ if

1) the process $x(t)$ is $F_{t}$-measurable for every $t \in \mathbf{R}$;

2) the trajectories of $x(t)$ are continuous with probability one;

3) for all $-\infty<t_{0}<t_{1}<\infty$,

$$
\sup _{t_{0} \leq t \leq t_{1}} \mathrm{E}|x(t)|^{2}<\infty
$$

4) for all $-\infty<t_{0}<t_{1}<\infty$,

$$
x(t)=x\left(t_{0}\right)+\int_{t_{0}}^{t} f(s, x(s)) d s+\int_{t_{0}}^{t} g(s, x(s)) d W(s)
$$

with probability one. (We understand the first integral in (2) as the Riemann integral, while the second is the Itô integral.)

We are interested in finding conditions for the existence of solutions of equation (1) that are mean square bounded or periodic (stationary) in the case where the equation is linear or weakly nonlinear. Without loss of generality we assume that $W(t)$ is onedimensional. The proof for the multidimensional case is analogous.

Many authors study this problem (see, for example, [1, 2], where one can find further references). The difference between the settings in those papers and the one used in

2000 Mathematics Subject Classification. Primary 34C25, 34C29, 34F05.

(C)2004 American Mathematical Society 
the current paper is that in [2], the matrix of the linear part is assumed to be constant or, if it is nonconstant, the equation is reduced to the matrix equation for the second moments. Rewriting the latter equation for every coordinate one obtains a system which has a higher order than the original system. Moreover, the conditions posed in [1, 2] on system (1) are given in terms of the system for the second moments. It is not easy to check those conditions, since the order of the system is quite large. Finally, the approach of [1,2] works only for linear systems and is not applicable to the case of weakly nonlinear systems.

We consider the case where the matrix of the linear part is varying. The conditions for the boundedness and periodicity of solutions are given in terms of the initial system, which is more convenient in practice.

In what follows we need an auxiliary result allowing one to differentiate stochastic Itô integrals with respect to a parameter. To make calculations simpler we treat only the one-dimensional case; however, the changes for the multidimensional case are obvious.

Lemma. Let a function $h(t, s)$ as well as its partial derivative $h_{t}^{\prime}(t, s)$ be continuous for all $t \geq s \in \mathbf{R}$. Assume that $f(t)$ is an $F_{t}$-measurable stochastic process such that

$$
\int_{-\infty}^{\infty}|h(t, s)|^{2} \mathrm{E}|f(s)|^{2} d s<\infty
$$

for all $t \in \mathbf{R}$. If the integral

$$
\int_{-\infty}^{t}\left|h_{t}^{\prime}(t, s)\right|^{2} \mathrm{E}|f(s)|^{2} d s
$$

converges uniformly with respect to $t \in\left[t_{1}, t_{2}\right]$, where $\left[t_{1}, t_{2}\right]$ is an arbitrary interval, then the stochastic integral

$$
y(t)=\int_{-\infty}^{t} h(t, s) f(s) d W(s)
$$

possesses the stochastic differential

$$
d y(t)=\left(\int_{-\infty}^{t} h_{t}^{\prime}(t, s) f(s) d W(s)\right) d t+h(t, t) f(t) d W(t) .
$$

Remark 1. The integral in (15) is understood as the mean square limit of the processes

$$
y_{n}(t)=\int_{-n}^{t} h(t, s) f(s) d W(s) .
$$

The limit exists by (3). The main properties of the ordinary Itô integrals hold for integrals of this kind, too (see, for example, [2] or [4]).

Remark 2. The latter result is obtained in [3 p. 264] for a finite interval.

Proof. It is necessary to prove that

$$
y\left(t_{2}\right)-y\left(t_{1}\right)=\int_{t_{1}}^{t_{2}}\left(\int_{-\infty}^{t} h_{t}^{\prime}(t, s) f(s) d W(s)\right) d t+\int_{t_{1}}^{t_{2}} h(t, t) f(t) d W(t)
$$

with probability one and for all $t_{1}<t_{2}$. Let $n>0$ be arbitrary and consider the proper stochastic integral

$$
y_{n}(t)=\int_{-n}^{t} h(t, s) f(s) d W(s)
$$

Condition (3) implies that

$$
\mathrm{E}\left|y_{n}(t)-y(t)\right|^{2} \rightarrow 0, \quad n \rightarrow \infty
$$


It follows from [3, p. 264] that

$$
y_{n}\left(t_{2}\right)-y_{n}\left(t_{1}\right)=\int_{t_{1}}^{t_{2}}\left(\int_{-n}^{t} h_{t}^{\prime}(t, s) f(s) d W(s)\right) d t+\int_{t_{1}}^{t_{2}} h(t, t) f(t) d W(t)
$$

almost surely for the sequence of stochastic processes $y_{n}(t)$, where $t_{1}<t_{2}$ are arbitrary numbers of the interval $\left[-n, t_{0}\right]$.

Fix arbitrary $t_{1}, t_{2} \in \mathbf{R}, n \in \mathbf{N}$, and choose $t_{0} \in \mathbf{R}$ such that $t_{1}, t_{2} \in\left[-n, t_{0}\right]$. Then

$$
\begin{gathered}
\mathrm{E}\left|y\left(t_{2}\right)-y\left(t_{1}\right)-\int_{t_{1}}^{t_{2}}\left(\int_{-\infty}^{t} h_{t}^{\prime}(t, s) f(s) d W(s)\right) d t-\int_{t_{1}}^{t_{2}} h(t, t) f(t) d W(t)\right|^{2} \\
=\mathrm{E} \mid y\left(t_{2}\right)-y_{n}\left(t_{2}\right)-y\left(t_{1}\right)+y_{n}\left(t_{1}\right)-\int_{t_{1}}^{t_{2}}\left(\int_{-\infty}^{t} h_{t}^{\prime}(t, s) f(s) d W(s)\right) d t \\
\quad-\int_{t_{1}}^{t_{2}} h(t, t) f(t) d W(t) \\
\quad+\int_{t_{1}}^{t_{2}}\left(\int_{-n}^{t} h_{t}^{\prime}(t, s) f(s) d W(s)\right) d t+\left.\int_{t_{1}}^{t_{2}} h(t, t) f(t) d W(t)\right|^{2} \\
\leq 3\left[\mathrm{E}\left|y\left(t_{2}\right)-y_{n}\left(t_{2}\right)\right|^{2}+\mathrm{E}\left|y\left(t_{1}\right)-y_{n}\left(t_{1}\right)\right|^{2}\right. \\
\quad+\mathrm{E} \mid \int_{t_{1}}^{t_{2}}\left(\int_{-\infty}^{t} h_{t}^{\prime}(t, s) f(s) d W(s)\right) d t \\
\left.-\left.\int_{t_{1}}^{t_{2}}\left(\int_{-n}^{t} h_{t}^{\prime}(t, s) f(s) d W(s)\right) d t\right|^{2}\right] .
\end{gathered}
$$

The first two terms in (11) approach zero as $n \rightarrow \infty$ by (9).

Now we estimate the last term in (11):

$$
\begin{aligned}
\mathrm{E} \mid \int_{t_{1}}^{t_{2}} & \left(\int_{-\infty}^{t} h_{t}^{\prime}(t, s) f(s) d W(s)\right) d t-\left.\int_{t_{1}}^{t_{2}}\left(\int_{-n}^{t} h_{t}^{\prime}(t, s) f(s) d W(s)\right) d t\right|^{2} \\
& =\mathrm{E}\left|\int_{t_{1}}^{t_{2}}\left(\int_{-\infty}^{-n} h_{t}^{\prime}(t, s) f(s) d W(s)\right) d t\right|^{2} \\
& \leq \mathrm{E}\left(\int_{t_{1}}^{t_{2}}\left|\int_{-\infty}^{-n} h_{t}^{\prime}(t, s) f(s) d W(s)\right| d t\right)^{2} \\
& \leq\left(t_{2}-t_{1}\right) \int_{t_{1}}^{t_{2}} \mathrm{E}\left|\int_{-\infty}^{-n} h_{t}^{\prime}(t, s) f(s) d W(s)\right|^{2} \\
& =\left(t_{2}-t_{1}\right) \int_{t_{1}}^{t_{2}}\left(\int_{-\infty}^{-n}\left(h^{\prime}\right)_{t}^{2}(t, s) \mathrm{E}|f(s)|^{2} d s\right) d t \\
& \leq\left(t_{2}-t_{1}\right)^{2} \sup _{t \in\left[t_{1}, t_{2}\right]} \int_{-\infty}^{-n}\left(h^{\prime}\right)_{t}^{2}(t, s) \mathrm{E}|f(s)|^{2} d s \rightarrow 0
\end{aligned}
$$

as $n \rightarrow \infty$. The latter relation holds, since integral (4) converges uniformly with respect to $t \in\left[t_{1}, t_{2}\right]$. Thus the right-hand side of (11) approaches zero as $n \rightarrow \infty$. This implies (7), whence the lemma follows.

Now we consider a linear nonhomogeneous system of Itô's stochastic equations of the form

$$
d x=[A(t) x+\alpha(t)]+\beta(t) d W(t)
$$


where $t \in \mathbf{R}, A(t)$ is a continuous matrix bounded on $\mathbf{R}$, and $\alpha$ and $\beta$ are continuous stochastic processes such that $\alpha(t)$ and $\beta(t)$ are $F_{t}$-measurable for every $t$ and

$$
\sup _{t \in \mathbf{R}} \mathrm{E}|\alpha(t)|^{2}<\infty, \quad \sup _{t \in \mathbf{R}} \mathrm{E}|\beta(t)|^{2}<\infty .
$$

A set of stochastic processes satisfying these conditions is denoted by $B$.

Then it is known (see, for example, 3, p. 234]) that there exists a solution of the Cauchy problem (12) with $x\left(t_{0}\right)=x_{0}$, where $x_{0}$ is an $F_{t_{0}}$-measurable random variable with a finite second moment. Moreover, the solution is unique for $t \geq t_{0}$ and possesses a finite second moment at every point $t \geq t_{0}$.

The following result gives conditions for the existence of solutions of equation (12) that are mean square bounded on the whole axis.

Theorem 1. If a deterministic system

$$
\frac{d x}{d t}=A(t) x
$$

is exponentially stable in the sense of [5, p. 251], then, whatever $\alpha(t)$ and $\beta(t) \in B$ are, system (12) has a unique solution that is mean square bounded on the whole axis. Moreover, this solution is exponentially stable in the mean square sense (see [6]).

Proof. Since the zero solution of system (14) is exponentially stable, for all $t \geq s$ there are positive constants $K$ and $\gamma$ such that

$$
\|\Phi(t, s)\| \leq K \exp \{-\gamma(t-s)\}
$$

where $\Phi(t, s)$ is the Cauchy matrix of system (14). Denote by $G(t, \tau)$ the Green function of system (14). The Green function is of the form

$$
G(t, s)= \begin{cases}\Phi(t, 0)(\Phi(s, 0))^{-1}, & t \geq s \\ 0, & t<s .\end{cases}
$$

The construction of the Green function and properties of fundamental matrices imply that $G(t, s)=\Phi(t, s)$ for $t \geq s$.

Let

$$
x^{*}=\int_{-\infty}^{t} \Phi(t, s) \alpha(s) d s+\int_{-\infty}^{t} \Phi(t, s) \beta(s) d W(s) .
$$

Both integrals in (17) exist, since

$$
\int_{-\infty}^{t}\|\Phi(t, s)\| \mathrm{E}|\alpha(s)| d s \leq \int_{-\infty}^{t} K \exp \{-\gamma(t-s)\} d s \sup _{t \in \mathbf{R}}\left(\mathrm{E}|\alpha(t)|^{2}\right)^{1 / 2}<\infty
$$

and

$$
\int_{-\infty}^{t}\|\Phi(t, s)\|^{2} \mathrm{E}|\beta(s)|^{2} d s \leq \int_{-\infty}^{t} K^{2} \exp \{-2 \gamma(t-s)\} d s \sup _{t \in \mathbf{R}} \mathrm{E}(|\beta(t)|)^{2}<\infty
$$

for arbitrary $t \in \mathbf{R}$. It is clear that $x^{*}$ is $F_{t}$-measurable. The second term in (17) is continuous, since

$$
\Phi(t, s)=\Phi(t, \tau) \Phi(\tau, s), \quad t \geq \tau \geq s,
$$

and the stochastic integral is represented in the following form:

$$
\begin{aligned}
\int_{-\infty}^{t} \Phi(t, s) \beta(s) d W(s) & =\int_{-\infty}^{a} \Phi(t, s) \beta(s) d W(s)+\int_{a}^{t} \Phi(t, s) \beta(s) d W(s) \\
& =\Phi(t, a) \int_{-\infty}^{a} \Phi(a, s) \beta(s) d W(s)+X(t) \int_{a}^{t} X^{-1}(s) \beta(s) d W(s),
\end{aligned}
$$


where $a \in \mathbf{R}$ is a fixed number and $X(t)$ is the fundamental matrix of the linear homogeneous part.

Now we show that $x^{*}(t)$ satisfies (12). Indeed, the first integral in (17) converges uniformly by (15) on every interval $\left[t_{1}, t_{2}\right]$ of the real axis. Its formal derivative also is uniformly convergent, since

$$
\frac{d}{d t} \Phi(t, s)=A(t) \Phi(t, s)
$$

and the matrix $A(t)$ is bounded. This justifies the differentiation procedure for the above stochastic integral.

Further, estimate (15) implies that

$$
\int_{-\infty}^{\infty}\|\Phi(t, s)\|^{2} \mathrm{E}|\beta(s)|^{2} d s<\infty
$$

and that the integral

$$
\int_{-\infty}^{t}\|\Phi(t, s)\|^{2} \mathrm{E}|\beta(s)|^{2} d s
$$

converges uniformly with respect to $t \in\left[t_{1}, t_{2}\right]$. Thus the stochastic differential for $x^{*}(t)$ can be obtained from the previous lemma:

$$
\begin{aligned}
d x^{*}(t)= & \left(\int_{-\infty}^{t} A(t) \Phi(t, s) \alpha(s) d s+\Phi(t, t) \alpha(t)\right) d t+\left(\int_{-\infty}^{t} A(t) \Phi(t, s) \beta(s) d W(s)\right) d t \\
& +\Phi(t, t) \beta(t) d W(t) \\
= & {\left[A(t)\left(\int_{-\infty}^{t} \Phi(t, s) \alpha(s) d s+\int_{-\infty}^{t} A(t) \Phi(t, s) \beta(s) d W(s)\right)+\alpha(t)\right] d t } \\
& +\beta(t) d W(t) \\
= & {\left[A(t) x^{*}(t)+\alpha(t)\right] d t+\beta(t) d W(t) . }
\end{aligned}
$$

The latter equality means that (17) satisfies system (12).

Finally we prove that $x^{*}(t)$ is bounded, by estimating every term in (17). It follows from the Cauchy-Bunyakovskiı inequality that

$$
\begin{aligned}
& \mathrm{E}\left|\int_{-\infty}^{t} \Phi(t, s) \alpha(s) d(s)\right|^{2} \leq \mathrm{E}\left(\int_{-\infty}^{t}\|\Phi(t, s)\| \cdot|\alpha(s)| d s\right)^{2} \\
& \quad \leq K^{2} \mathrm{E}\left(\int_{-\infty}^{t} \exp \left\{-\frac{\gamma}{2}(t-s)\right\} \exp \left\{-\frac{\gamma}{2}(t-s)\right\}|\alpha(s)| d s\right)^{2} \\
& \quad \leq K^{2} \int_{-\infty}^{t} \exp \{-\gamma(t-s)\} d s \int_{-\infty}^{t} \exp \{-\gamma(t-s)\} \mathrm{E}|\alpha(s)|^{2} d s \\
& \quad \leq K^{2} \frac{1}{\gamma^{2}} \sup _{t \in \mathbf{R}} \mathrm{E}|\alpha(t)|^{2}<\infty .
\end{aligned}
$$

Properties of stochastic integrals imply that

$$
\begin{aligned}
\mathrm{E}\left|\int_{-\infty}^{t} \Phi(t, s) \beta(t) d W(s)\right|^{2} & \leq \int_{-\infty}^{t} K^{2} \exp \{-2 \gamma(t-s)\} \sup _{t \in \mathbf{R}} \mathrm{E}|\beta(s)|^{2} d s \\
& \leq K^{2} \frac{1}{2 \gamma} \sup _{t \in \mathbf{R}} \mathrm{E}|\beta(t)|^{2}<\infty .
\end{aligned}
$$

Applying the latter relations and boundedness of the second moments of $\alpha(t)$ and $\beta(t)$ we obtain

for some constant $C>0$.

$$
\sup _{t \in \mathbf{R}} \mathrm{E}\left|x^{*}(t)\right|^{2} \leq C
$$


Let us show that $x^{*}(t)$ is a mean square exponentially stable solution. Let $x(t)$ be an arbitrary solution of system (12) such that $x(0)=x_{0}$, where $x_{0}$ is an $F_{0}$-measurable random variable with $\mathrm{E}\left|x_{0}\right|^{2}<\infty$. Then

$$
x(t)=\Phi(t, 0) x_{0}+\int_{0}^{t} \Phi(t, s) \alpha(s) d s+\int_{0}^{t} \Phi(t, s) \beta(s) d W(s)
$$

for $t \geq 0$. This representation can be proved by evaluating the stochastic differential and applying the uniqueness of the solution.

Thus

$$
\mathrm{E}\left|x(t)-x^{*}(t)\right|^{2} \leq\|\Phi(t, 0)\|^{2} \mathrm{E}\left|x^{*}(0)-x_{0}\right|^{2} \leq K^{2} \exp \{-2 \gamma t\} \mathrm{E}\left|x^{*}(0)-x_{0}\right|^{2},
$$

whence the exponential stability follows.

Now we prove that $x^{*}(t)$ is strongly unique.

Let $y(t)$ be a solution of system (12) that is different from $x^{*}(t)$ and is mean square bounded on the whole axis. Then $z(t)=x^{*}(t)-y(t)$ is a solution of system (14). Since this system is exponentially stable, $z(t)$ is such that

$$
|z(t)| \leq K \exp \{\gamma(t-\tau)\}|z(\tau)|
$$

with probability one for all $t \geq \tau$. It is obvious that $\sup _{t \in \mathbf{R}} \mathrm{E}|z(t)|^{2}=a<\infty$.

Thus

$$
\mathrm{E}|z(t)|^{2} \leq K^{2} \exp \{-2 \gamma(t-\tau)\} a
$$

for all $t, \tau \in \mathbf{R}$ such that $t \geq \tau$. Passing to the limit in the latter inequality as $\tau \rightarrow-\infty$ we obtain $\mathrm{E}|z(t)|^{2}=0$ for all $t \in \mathbf{R}$, whence it follows that

$$
\mathrm{P}\left\{x^{*}(t) \neq y(t)\right\}=0 \text { for all } t \in \mathbf{R} .
$$

Since $x^{*}(t)$ and $y(t)$ are continuous, we have proved the strong uniqueness of the solution. Therefore

$$
\mathrm{P}\left\{\sup _{t \in \mathbf{R}}\left|x^{*}(t)-y(t)\right|>0\right\}=0 .
$$

The uniqueness is proved, and this completes the proof of the theorem.

Now we turn to the problem on the existence of periodic solutions of system (12).

Let $A(t)$ be a matrix periodic in $t$ with period $T$. Assume that $\alpha(t)$ and $\beta(t)$ are such that

$$
\eta(t)=(\alpha(t), \beta(t))
$$

is periodic in the weak sense (that is, its finite-dimensional distributions are periodic with period $T$ ). The process $x^{*}(t)$ defined by (17) is a $T$-periodic solution of system (12).

Indeed, the matrix $A(t)$ is $T$-periodic and thus the Cauchy matrix of system (14) satisfies

$$
\Phi(t+T, s+T)=\Phi(t, s)
$$

since

$$
\begin{gathered}
\Phi(t+T, s+T)=\Phi(t+T, 0)\left(\Phi(s+T, 0)^{-1}=\Phi(t, 0) \Phi(T, 0)\right. \\
(\Phi(T, 0))^{-1}(\Phi(s, 0))^{-1}=\Phi(t, s)
\end{gathered}
$$

by properties of fundamental matrices. We show that every term in (17) is a $T$-periodic stochastic process.

Indeed, the first term is $T$-periodic by (15) and (19) and by property $7^{0}$ in [2, p. 184]. The second term in (17) is also periodic, since so is the integral

$$
\eta_{n}(t)=\int_{t-n}^{t} \Phi(t, s) \beta(s) d W(s)
$$


for all $n \geq 1$. Changing the variable we get

$$
\eta_{n}(t)=\int_{-n}^{0} \Phi(t, t+s) \beta(t+s) d W(t+s)
$$

The rest of the proof is the same as that in 2 p. 186] upon observation that $\beta(t+s)$ is periodic in $t$ as well as the function $\Phi(t, t+s)$ is, in view of (19)).

Therefore both processes on the right-hand side of (17) are periodic, whence $x^{*}(t)$ is $T$-periodic, since $\alpha(t)$ and $\beta(t)$ are periodically related.

Thus we have proved the following corollary of Theorem 1.

Corollary 1. Assume that the matrix $A(t)$ in system (12) is T-periodic and the process $\eta(t)=(\alpha(t), \beta(t))$ is T-periodic in the weak sense. If all the assumptions of Theorem 1 hold, then the solution $x^{*}(t)$ defined by (17) is a stochastic process $T$-periodic in the weak sense. Moreover, if the matrix $A(t)$ is constant and the process $\eta(t)$ is stationary in the weak sense, then the solution $x^{*}(t)$ is stationary.

Equation (12) can be viewed as a result of perturbation of the linear system (14) by random forces $\alpha(t) d t+\beta(t) d W(t)$. Now we consider a more general case where these forces are nonlinear, namely we consider the following stochastic system:

$$
d x=[A(t) x+f(t, x)] d t+g(t, x) d W(t)
$$

where $A(t)$ is a continuous and bounded matrix on $\mathbf{R}$, and the functions $f(t, x)$ and $g(t, x)$ are defined and continuous for $t \in \mathbf{R}$ and $x \in \mathbf{R}^{n}$. Moreover, we assume that these functions satisfy the Lipschitz condition in $x \in \mathbf{R}^{n}$ with a constant $L$. We also assume that the functions $f(t, 0)$ and $g(t, 0)$ are bounded on $\mathbf{R}$ by some constant $N$. Under these conditions the following result holds.

Theorem 2. If system (14) is exponentially stable and the constant $L$ is such that

$$
\frac{K^{2} L^{2}}{\gamma}+K^{2} L^{2}-\gamma<0
$$

then system (20) has a unique mean square bounded solution. Moreover, this solution is mean square exponentially stable.

If additionally the functions $f$ and $g$ are periodic in $t$ with period $T$, then the solution is $T$-periodic.

Finally if $A, f$, and $g$ do not depend on $t$, then the solution is stationary.

Proof. We apply the successive approximation method to equation (20). We seek its bounded solution as the limit of the sequence $\left\{x_{m}(t)\right\}$, where $\left\{x_{m+1}(t)\right\}$ is defined as a unique mean square bounded solution of the linear system

$$
d x=\left[A(t) x+f\left(t, x_{m}(t)\right)\right] d t+g\left(t, x_{m}(t)\right) d W(t), \quad x_{0}(t)=0 .
$$

By Theorem 1, each of these systems has a unique solution bounded on the axis, since

$$
\begin{aligned}
& \mathrm{E}\left|f\left(t, x_{m}(t)\right)\right|^{2} \leq 2\left(L^{2} M\left|x_{m}(t)\right|^{2}+N^{2}\right), \\
& \mathrm{E}\left|g\left(t, x_{m}(t)\right)\right|^{2} \leq 2\left(L^{2} M\left|x_{m}(t)\right|^{2}+N^{2}\right),
\end{aligned}
$$

and $x_{m}(t)$ is $F_{t}$-measurable, whence the nonhomogeneous term on the right-hand side of (21) belongs to the class $B$. Theorem 1 also implies that the solution is of the form

$$
x_{m+1}(t)=\int_{-\infty}^{t} \Phi(t, s) f\left(s, x_{m}(s)\right) d s+\int_{-\infty}^{t} \Phi(t, s) g\left(s, x_{m}(s)\right) d W(s) .
$$

Similarly to [2], one can prove that the solutions $x_{m}(t)$ converge with probability one uniformly on an arbitrary interval $\left[t_{1}, t_{2}\right]$, and that

$$
\mathrm{E}\left|x_{m}(t)\right|^{2} \leq C
$$


for all $t \in \mathbf{R}$ and some $C>0$. By Fatou's lemma this implies that

$$
\mathrm{E}\left|x_{\infty}(t)\right|^{2} \leq C .
$$

Passing to the limit in (22) as $m \rightarrow \infty$ and using properties of stochastic integrals and the continuity of the functions $f$ and $g$ we prove that the limit stochastic process $x_{\infty}(t)$ is such that

$$
d x_{\infty}(t)=\int_{-\infty}^{t} \Phi(t, s) f\left(s, x_{\infty}(s)\right) d s+\int_{-\infty}^{t} \Phi(t, s) g\left(s, x_{\infty}(s)\right) d W(s) .
$$

Differentiating it and applying the lemma we show that the limit process $x_{\infty}(t)$ satisfies system (20). The $F_{t}$-measurability is obvious, since $x_{\infty}(t)$ is the limit of $F_{t}$-measurable processes. The uniqueness of such a process can be proved by the same argument as in [2] p. 272].

The solution obtained this way is exponentially stable. Indeed, let $y(t)$ be another solution of system (20) in the sense explained before. As in [2] p. 273] we prove that

$$
y(t)=\Phi(t, 0) y(0)+\int_{0}^{t} \Phi(t, s) f(s, y(s)) d s+\int_{0}^{t} \Phi(t, s) g(s, y(s)) d W(s) .
$$

It is seen from (23) that the process $x_{\infty}(t)$ satisfies an analogous relation.

Subtracting we obtain

$$
\begin{aligned}
& \mathrm{E}\left|x_{\infty}(t)-y(t)\right|^{2} \\
& \leq 3\left[K \exp \{-\gamma t\} \mathrm{E}\left|x_{\infty}(0)-y(0)\right|^{2}\right. \\
& \quad+\frac{K^{2} L^{2}}{\gamma} \int_{0}^{t} \exp \{\gamma(t-s)\} \mathrm{E}\left|x_{\infty}(s)-y(s)\right|^{2} d s \\
& \left.\quad+K^{2} L^{2} \int_{0}^{t} \exp \{\gamma(t-s)\} \mathrm{E}\left|x_{\infty}(s)-y(s)\right|^{2} d s\right]
\end{aligned}
$$

or

$$
u(t) \leq 3\left[K \mathrm{E}\left|x_{\infty}(0)-y(0)\right|^{2}+\left(\frac{K^{2} L^{2}}{\gamma}+K^{2} L^{2}\right) \int_{0}^{t} u(s) d s\right],
$$

where $u(t)=\exp \{\gamma t\} \mathrm{E}\left|x_{\infty}(t)-y(t)\right|^{2}$, whence by the Gronwall-Bellman inequality

$$
u(t) \leq 3 K \mathrm{E}\left|x_{\infty}(0)-y(0)\right|^{2} \exp \left\{\left(\frac{K^{2} L^{2}}{\gamma}+K^{2} L^{2}\right) t\right\}
$$

or

$$
\mathrm{E}\left|x_{\infty}(t)-y(t)\right|^{2} \leq 3 K \mathrm{E}\left|x_{\infty}(0)-y(0)\right|^{2} \exp \left\{\left(\frac{K^{2} L^{2}}{\gamma}+K^{2} L^{2}-\gamma\right) t\right\} .
$$

The assumption on the Lipschitz constant implies by inequality (25) that $x_{\infty}(t)$ is exponentially stable.

The proof of the remaining parts of the theorem concerning periodic and stationary solutions is based on the following observation. Theorem 1 implies that $x_{\infty}(t)$ is the limit of a sequence of periodic (stationary) processes $x_{m}(t)$ defined by nonhomogeneous system (21). The existence of a second periodic (stationary) solution of system (20) contradicts the uniqueness of a mean square bounded solution of this system, since every periodic (stationary) solution has bounded second moments. The theorem is proved. 


\section{BIBLIOGRAPHY}

1. L. Ruifeld and V. Mandekar, Stochastic semilinear evolution equations: Lyapunov function, stability, and ultimate boundedness, J. Math. Anal. Appl. 12 (1998), no. 2, 98-115.

2. A. Ya. Dorogovtsev, Periodic and Stationary Regimes of Infinite Dimensional Deterministic and Stochastic Dynamic Systems, "Vyshcha shkola", Kiev, 1992. (Russian) MR 94c:60097

3. E. F. Tsar'kov, Random Disturbances of Functional-Differential Equations, "Zinatne", Riga, 1989. (Russian) MR 90m:34164

4. R. Sh. Liptser and A. N. Shiryaev, Theory of Martingales, "Nauka", Moscow, 1974; English transl., Kluwer, Dordrecht, 1989. MR 90j:60046

5. P. P. Demidovich, Lectures on the Mathematical Theory of Stability, "Nauka", Moscow, 1967. (Russian) MR 37:1716

6. R. Z. Khas'minskiū, Stability of Systems of Differential Equations Under Random Perturbations of Their Parameters, "Nauka", Moscow, 1969; English transl., Sijthoff \& Noordhoff, Alphen aan Rijn, 1980. MR 41:3925

Faculty for Mechanics and Mathematics, Kyiv National Taras Shevchenko University, Volodymyrs'Ka Street 64, Kyiv 01033, Ukraine

E-mail address: stom@mail.univ.kiev.ua

Received 1/MAY/2001

Translated by V. SEMENOV 